\title{
Long-term variability in the X-ray emission of RX J0720.4-3125
}

\author{
C. P. de Vries ${ }^{1}$, J. Vink ${ }^{1}$, M. Méndez ${ }^{1}$, and F. Verbunt ${ }^{2}$ \\ 1 SRON, National Institute for Space Research, Sorbonnelaan 2, 3584 CA Utrecht, The Netherlands \\ 2 Astronomical Institute, Utrecht University, PO Box 80000, 3508 TA Utrecht, The Netherlands
}

Received 28 December 2003 / Accepted 14 January 2004

\begin{abstract}
We detect a gradual, long-term change in the shape of the X-ray spectrum of the isolated neutron star RX J0720.4-3125, such that the spectrum of the source can no longer be described as a blackbody spectrum. The change is accompanied by an energy-dependent change in the pulse profile. If the X-ray emission is influenced by the magnetic field of the pulsar, these changes in spectral shape may point to precession of the neutron star.
\end{abstract}

Key words. neutron stars - X-rays

\section{Introduction}

RX J0720.4-3125 (Haberl et al. 1997) belongs to the group of radio-quiet neutron stars discovered by ROSAT. As these objects are characterised by soft X-ray emission from the surface, it was hoped that high resolution spectroscopy of those sources with the Chandra's Low Energy Grating Spectrometer (LETGS) or XMM-Newton's Reflection Grating spectrometer (RGS) would reveal line features from a thin atmosphere that could provide information on the surface gravity of the neutron star and hence on the equation of state of the neutron star matter. However, deep observations with XMM-Newton and Chandra of RX J0720.4-3125 (Paerels et al. 2001; Kaplan et al. 2003) and RX J1856.5-3754 (Burwitz et al. 2001) have in that respect been somewhat of a disappointment, as the spectra are almost perfectly well described by a blackbody spectrum. The only unusual feature of the X-ray spectra is that the blackbody emission implies a radius too small for a neutron star and, moreover, underpredicts the observed optical and UV flux (Kaplan et al. 2003; Burwitz et al. 2001; Motch et al. 2003). This implies that another cooler component is present and that the X-ray emission only comes from part of the surface.

RX J0720.4-3125 has a low absorbing column density $\left(N_{\mathrm{H}} \approx 10^{20} \mathrm{~cm}^{-2}\right)$, is at an approximate distance of $\approx 300 \mathrm{pc}$ (Kaplan et al. 2003), and shows sinusodial pulsations with a period of $8.39 \mathrm{~s}$ (Haberl et al. 1997; Cropper et al. 2001). The upper limit to the period derivative $\dot{P}<n \times 10^{-13} \mathrm{ss}^{-1}$, with $n$ "a few" (Kaplan et al. 2002), implies a magnetic field strength $B<3 \sqrt{n} \times 10^{13} \mathrm{G}$ and a characteristic age $\tau_{\mathrm{c}} \equiv 0.5 P / \dot{P}>$ (1.3/n) Myr. As RX J0720.4-3125 was considered to be a perfect blackbody spectrum with a temperature of $k T_{\mathrm{BB}} \approx 86 \mathrm{eV}$ it was observed several times by XMM-Newton for calibration

Send offprint requests to: C.P. de Vries, e-mail: C.Pde.Vries@sron.nl
Table 1. XMM-Newton observations of RX J0720.4-3125.

\begin{tabular}{ccccc}
\hline \hline Orbit & Observation & Date & Julian date & Duration (ks) \\
\hline 0078 & 0124100101 & $13-05-2000$ & 2451677.9 & 65 \\
0175 & 0132520301 & $21-11-2000$ & 2451870.3 & 30 \\
0533 & 0156960201 & $06-11-2002$ & 2452585.4 & 30 \\
0534 & 0156960401 & $08-11-2002$ & 2452498.5 & 32 \\
0622 & 0158360201 & $02-05-2003$ & 2452762.5 & 81 \\
0711 & 0161960201 & $27-10-2003$ & 2452940.5 & 45 \\
\hline
\end{tabular}

purposes. However, as we will demonstrate here, the spectrum of RX J0720.4-3125 has slowly hardened and cannot be described any more by a blackbody spectrum, while the pulse shape has become narrower and the phase dependence of the spectrum changed with time.

While we were finalising this paper, Haberl et al. (2003b) released a pre-print in which they show that the spectrum of RX J0720.4-3125 depends on the pulse phase. They also discuss changes in the spectrum as found with EPIC, but assign these to calibration inaccuracies.

\section{Observations}

Table 1 gives the $\log$ of XMM-Newton observations of RX J0720.4-3125. Since the source spectrum is rather soft, and most of the emission comes from below $\sim 1.8 \mathrm{keV}$, we use RGS (den Herder et al. 2001) data for the spectral analysis; due to the nature of the gratings, the effective area of the RGS is rather insensitive to changes in CCD gain and charge transfer inefficiency. The RGS was operated in normal spectroscopic mode, yielding a time resolution of $4 \mathrm{~s}$, making it unsuitable for a proper timing analysis. Therefore, for this purpose we use EPIC/PN (Strüder et al. 2001) since it has the largest effective area among the instruments on board XMM-Newton, and operates in a high-time resolution mode. 


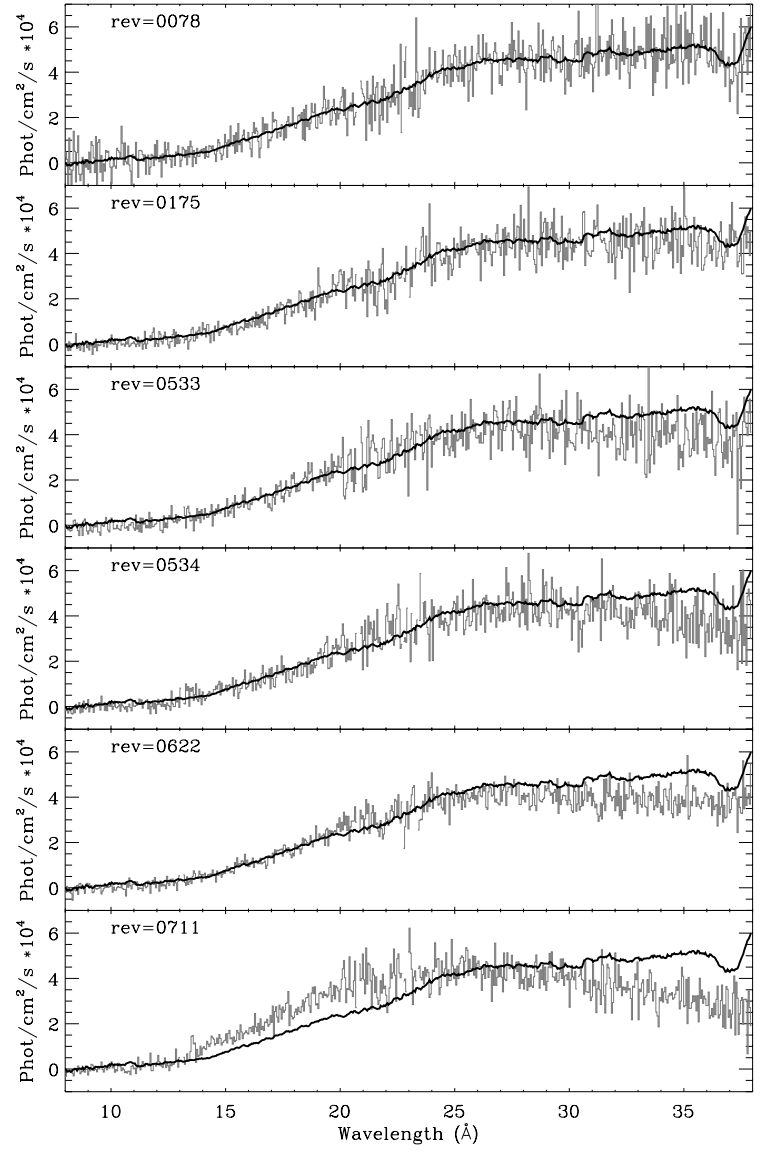

Fig. 1. Fluxed RGS spectra of the different observations of RX J0720.4-3125. The spectra are shown as the light grey histograms. The black lines are the smoothed data from orbit 0078. The differences between the lines and the different data show the variations of the spectrum with respect to the data of orbit 0078 . Especially the large change at orbit 0711 with an increase of flux between 15 and $25 \AA$ can be clearly recognized.

During the first four observations, PN was operated in fullframe mode, which produces an image of $378 \times 384$ pixels with a time resolution of $73.4 \mathrm{~ms}$. During the last two observations the PN was switched to small-window mode, yielding images of $63 \times 64$ pixels with a time resolution of $6 \mathrm{~ms}$. The thin filter was used in revolutions 0078,0533 , and 0534 , the medium one in revolution 0175 , and the thick one in revolution 0622. The observation of revolution 0711 started with the thin filter, and later switched to the medium one. All data were processed using the standard XMM-Newton software SAS version 5.4.1.

\subsection{Spectral analysis}

Figure 1 shows the RGS spectra for the 6 revolutions. The indivual RGS's agree with each other within the statistical uncertainties, so the data from both RGS's have been combined. From the figure it is apparent that the spectrum of RX J0720.43125 gradually changes, while the total flux appears to remain fairly constant. The change of the spectrum is most noticeable in orbit 0711 , where there is an increase of the flux in the $15 \AA$ to $25 \AA$ range, and a drop in the flux above $30 \AA$.
To verify that the RGS instrument is stable and that its effective area does not change, we have also analyzed data from two sources that are stable on a time scale of years.

The first source is the supernova remnant 1E 0102-72.3 in the Small Magellanic Cloud, a calibration source that is regularly observed with the RGS. From the strength of the bright emission lines from this source in the range 13 to $23 \AA$, as measured in data from orbits 0065 to 0711 , we find that the effective area of the instrument in this range changes by less than $3 \%$. In the long wavelength range the SNR 0102-72.3 spectrum has a weak carbon line at $33.7 \AA$. The upper limit on possible changes in the effective area of the RGS instrument at this wavelength is about $10 \%$, mainly determined by the limited statistics in this line. All data from 1E 0102-72.3 are consistent with no change in the RGS effective area. The second source is the calibration source Mrk 421. An upper limit to any change in the neutral Oxygen absorption edge around $22.8 \AA$, which is a tracer of possible instrument contamination (e.g. ice), is $5 \%$.

Since all upper limits on possible changes in effective area are well below the changes seen in RX J0720.4-3125 we conclude that the X-ray spectrum of RX J0720.4-3125 itself is subject to change over the course of about 3 years.

For the spectral analysis we use the XSPEC spectral fitting package (Arnaud 1996). As noted by Paerels et al. (2001) the data of orbit 0078 can be well fitted by an absorbed blackbody spectrum. With the improved calibration of the RGS we find a temperature of $k T_{\mathrm{BB}}=86.7 \pm 0.3 \mathrm{eV}$ and an interstellar column density of $N_{\mathrm{H}}=(1.41 \pm 0.07) \times 10^{20} \mathrm{~cm}^{-2}$, in reasonable agreement with the values obtained with the Chandra LETGS instrument, $k T_{\mathrm{BB}}=81.4 \pm 1.3 \mathrm{eV}$ and $N_{\mathrm{H}}=(1.32 \pm 0.14) \times 10^{20} \mathrm{~cm}^{-2}$, by Kaplan et al. (2003). The LETGS observation was made in February 2000, four months before the first XMM-Newton observation.

Fits with the same model to the remaining spectra yield a temperature increase with time, as well as changes as large as $\Delta N_{\mathrm{H}} \sim 5 \times 10^{20} \mathrm{~cm}^{-2}$ in the interstellar absorption. The model, however, does not provide satisfactory fits to all the spectra.

Next, we fit all the data simultaneously. Since large variations of the interstellar absorption over such a short period are unlikely, we assume that $N_{\mathrm{H}}$ remains constant; we therefore couple this parameter for all observations, while we let the blackbody temperature and normalization vary freely between observations (Table 2). Although these fits serve to emphasize the gradual hardening of the spectra, half of the spectra, especially that of revolution 0711 , are poorly fit by this model.

Following Haberl et al. (2003a) and van Kerkwijk (2004), next we fit the data with a model that consists of a blackbody affected by additional absorption by a broad Gaussian line, as might be expected in the case of cyclotron absorption. In these fits, we constrain the parameters of the blackbody model to be the same for all the observations, whereas the parameters of the line are allowed to vary independently. Although this model fits the data well, in most cases we find that the width of the line is larger than its central energy, or the central energy of the line lies outside the spectral range covered by the data. In those cases, the tail of the broad Gaussian just serves to attenuate the emission of the source at long wavelengths. Furthermore, we find no clear trend in the central energy or the width of the line. 
Table 2. Spectral fits ${ }^{a}$ to the observations of RX J0720.4-3125.

\begin{tabular}{ccccccc}
\hline \hline Orbit & $k T(\mathrm{eV})$ & $L_{\mathrm{bb}}^{b}$ & $\Gamma^{c}$ & \multicolumn{3}{c}{ Flux $^{d}$} \\
& & & & $(10-23 \AA)$ & $(23-38 \AA)$ & $(10-38 \AA)$ \\
\hline 0078 & $81.3(3)$ & $2.54(3)$ & $1.44(5)$ & $1.86(4)$ & $4.72(7)$ & $6.58(10)$ \\
0175 & $81.4(5)$ & $2.42(3)$ & $1.45(5)$ & $1.77(5)$ & $4.49(7)$ & $6.26(11)$ \\
0533 & $84.6(4)$ & $2.22(3)$ & $1.74(5)$ & $1.89(4)$ & $4.20(7)$ & $6.09(11)$ \\
0534 & $84.0(4)$ & $2.28(3)$ & $1.67(5)$ & $1.88(4)$ & $4.31(7)$ & $6.19(11)$ \\
0622 & $86.3(3)$ & $2.07(2)$ & $1.85(6)$ & $1.93(4)$ & $4.08(7)$ & $6.01(06)$ \\
0711 & $98.2(4)$ & $1.96(2)$ & $2.70(8)$ & $2.81(4)$ & $4.01(6)$ & $6.82(09)$ \\
\hline
\end{tabular}

${ }^{a}$ Fit range 10-38 A. For the interstellar absorption component we use cross sections from Verner et al. (1996), and abundances from Wilms et al. (2000). Numbers in parentheses are 1- $\sigma$ confidence limits in the last digit(s). For the fits with a blackbody, the best-fit value for $N_{\mathrm{H}}$, constrained to be the same in all observations, is $(4.3 \pm 0.1) \times 10^{20} \mathrm{~cm}^{-2}$. For the fits with a blackbody times a power law, the bestfit values for $N_{\mathrm{H}}, k T$, and the bolometric luminosity of the blackbody, constrained to be the same in all observations are, respectively, $(1.28 \pm 0.1) \times 10^{20} \mathrm{~cm}^{-2}, 70.3 \pm 0.1 \mathrm{eV}$, and $(3.04 \pm 0.02) \times 10^{32} d_{300}^{2} \mathrm{erg} \mathrm{s}^{-1}$, with $d_{300}$ the distance to RX J0720.4-3125 in units of $300 \mathrm{pc}$. For the fits with a blackbody model, $\chi^{2}=874.1$ for 662 degrees of freedom. For the fits with a blackbody times a power-law model $\chi^{2}=776.8$ for 668 degrees of freedom.

${ }^{b}$ Bolometric luminosity of the blackbody in units of $10^{32} d_{300}^{2} \mathrm{erg} \mathrm{s}^{-1}$, where $d_{300}$ is the distance to the source in units of $300 \mathrm{pc}$.

${ }^{c}$ Index of the power law in the model that consists of a blackbody times a power law (see text).

$d$ Observed flux, obtained from the fits with a blackbody times a power-law; only statistical errors are indicated. The systematic errors in the flux are of order 5-10\%.

We find that the ratio of the spectra of the last and the first observation is close to a power law. We therefore fit the data to an empirical model that consists of a blackbody multiplied by a power law $E^{\Gamma}$, all affected by interstellar absorption. While the index of the multiplicative power law is allowed to change between observations, for these fits we constrain the parameters of the blackbody and the interstellar absorption to be the same in all observations. While it is difficult to assign a physical interpretation to this model, it provides an acceptable description of the data in the RGS range (10-38 $\AA$, see Table 2), it has fewer parameters than the Gaussian absorption model and, in addition, the index of the power law increases steadily over the course of the observations.

\subsection{Timing analysis}

Starting from the raw data, we first produce a list of calibrated events. To reduce pile-up, in the next step we select only single events as well as events that are not affected by some of the imperfections (bad columns, hot pixels, etc.) of the CCDs. We extract events within a 39 arcsec circle centred on the source. We barycenter these events using the SAS routine BARYCEN version 1.13.4, and we then separate the events according to their energy in 3 event lists; the bands that we use are 0.1 to $1.2 \mathrm{keV}, 0.1$ to $0.4 \mathrm{keV}$, and 0.4 to $0.8 \mathrm{keV}$, respectively.

For each observation we find the best period in the full band using an epoch folding technique; in all cases we find a period of $8.391 \mathrm{~s}$, consistent with the value previously found for this source by Kaplan et al. (2003). We then produce folded light curves in the three bands, and we also compute a folded hardness-ratio light curve from the ratio of the $0.4-0.8 \mathrm{keV}$ and the $0.1-0.4 \mathrm{keV}$ light curves. In Fig. 2 we show the $0.1-1.2 \mathrm{keV}$ and the hardness-ratio light curves. For each observation we define the phase such that the maximum of the full-band light

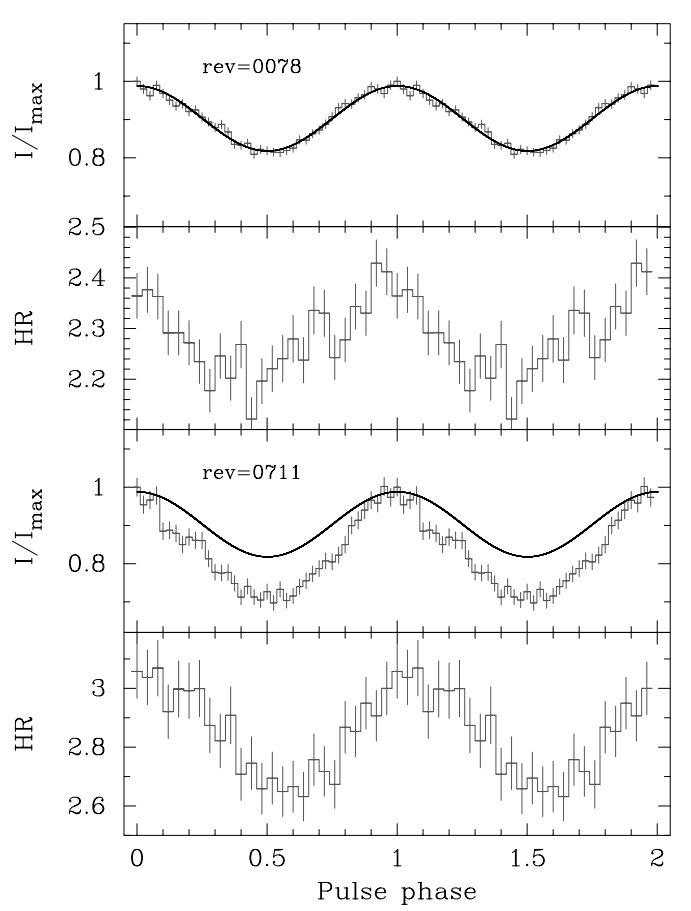

Fig. 2. Pulse profile of RX J0720.4-3125 in the 0.1-1.2 keV band (panels 1 and 3 from top to bottom) and hardness-ratio light curve (panels 2 and 4) for revolutions 0078 and 0711 using EPIC/PN. The 0.1-1.2 keV light curves are normalised to 1 at the maximum. Phase 0 is defined when the full-band light curve reaches its maximum. The solid line is the best sinusoidal fit to the full-band light curve of revolution 0078 . The same sinusoidal fit is overplotted on top of the full-band light curve of the other revolution for comparison. For both observations the thin filter was used.

curve occurs at phase zero; the phase of the hardness ratio light curves is the same as for the full-band light curves.

The pulse profile in the $0.1-1.2 \mathrm{keV}$ band, as well as the hardness-ratio pulse profile, change from one observation to 
the other. The first panel in Fig. 2 shows a sinusoidal fit to the pulse profile during the first observation; the same sine function is overplotted to the full-band pulse profiles obtained from the other observations. It is apparent that the pulse profile becomes narrower with time.

At the same time, the hardness-ratio pulse profile also changes. In the first observation there is a clear modulation, and the hardness-ratio profile leads the full-band light curve by $0.064 \pm 0.017$ in phase. In the following observations the amplitude of the hardness-ratio modulation decreases and the phase difference between the full-band and the hardness-ratio light curves is consistent with zero. Eventually, in the last observation the modulation increases again, but now the hardness-ratio light curve lags the full band-light curve by $-0.126 \pm 0.010$ in phase.

\section{Conclusions and discussion}

The XMM-Newton data of RX J0720.4-3125 show that the spectrum of the source changes on a time scale of years, the first time ever that the X-ray spectrum of an isolated neutron star, other then soft gamma-ray repeaters or anomalous X-ray pulsars, is seen to change. Whereas the changes are most pronounced in the last observation, we think that the actual change is gradual, as witnessed by a gradual increase in the temperatures derived from the blackbody fits; or by a gradual increase in the index of the powerlaw in the fits with a blackbody multiplied with a power law (Table 2 and Fig. 1). The spectral changes are accompanied by an energy-dependent change in the pulse shape; in particular the pulse phase where the spectrum is hardest has moved with respect to the phase of maximum flux (Fig. 2).

The phase (i.e. angle) dependent spectrum of single neutron stars is currently not explained. The broad absorption features have been interpreted as a proton-cyclotron absorption feature (Haberl et al. 2003a). In pulsars with a strong field (probably stronger than the limit for RX J0720.4-3125) the absorption feature may be weakened by the strong-field quantum electrodynamics effect of vacuum resonance mode conversion (Lai \& Ho 2003). The neutron star spectra have also been interpreted as due to cyclotron-resonance scattering of the spectrum from the surface of the neutron star, by electron-positron pairs in the magnetosphere (Ruderman 2003). For both interpretations the spectrum is likely to be angle and energy dependent, in accordance with the variation of the X-ray spectrum (as measured by hardness ratio) with pulse phase.

To explain the gradual, long-term variation we consider two general possibilities: either the intrinsic spectrum of the neutron star changes, or our view of the neutron star changes. The intrinsic spectrum of the neutron star could change, due to energy release deep in the neutron star, due to a glitch for example, causing the surface to gradually become hotter. We consider this unlikely as the explanation for the changing spectrum of RX J0720.4-3125, because it does not explain the change in pulse shape. Also this model would predict the total flux to increase with the temperature, in contrast to what is observed. Another possibility, valid for Ruderman's model, would be that the electron-positron plasma surrounding the neutron star changes. So far, there is no specific prediction in the Ruderman model for changes in the magnetospheric plasma on a year-long time scale.

Therefore, we suggest that the variation in the spectrum of RX J0720.4-3125 is caused by a change in the angle under which we see the emitting region and/or the covering electronpositron plasma, caused by precession of the neutron star. Precession arises when the form of the neutron star deviates from a perfect sphere and its rotation is not around a principal axis (as reviewed by e.g. Link 2003). The changes in pulse form and phase of the radio pulsar B1828-11 are successfully described with free precession, and have a time scale of years (Stairs et al. 2000), i.e. comparable to the time scale on which the variations in the spectrum and pulses of RX J0720.4-3125 occur. Whether a change in viewing angle can produce the observed hardening of the spectrum, with only a modest change in overall flux, will depend on the details of the emission model, and requires further investigation.

To further investigate the cause of the long-term variability it will be useful to investigate the phase-dependence of the spectra in more detail; to obtain a reliable period derivative of the pulses, and look for the phase changes expected from precession; and finally to see whether the changes continue.

Acknowledgements. The authors whish to thank Frank Haberl for a very useful discussion on the effect of EPIC-pn observation modes and Jan-Willem den Herder for his continuous stimulation.

\section{References}

Arnaud, K. A. 1996, in Astronomical Data Analysis Software and Systems V, 5, ASP Conf. Ser., 101, 17

Burwitz, V., Zavlin, V. E., Neuhäuser, R., et al. 2001, A\&A, 379, L35

Cropper, M., Zane, S., Ramsay, G., Haberl, F., \& Motch, C. 2001, A\&A, 365, L302

den Herder, J. W., Brinkman, A. C., Kahn, S. M., et al. 2001, A\&A, 365, L7

Haberl, F., Motch, C., Buckley, D. A. H., Zickgraf, F.-J., \& Pietsch, W. 1997, A\&A, 326, 662

Haberl, F., Schwope, A. D., Hambaryan, V., Hasinger, G., \& Motch, C. 2003a, A\&A, 403, L19

Haberl, F., Zavlin, V. E., Truemper, J., \& Burwitz, V. 2003b [astro-ph/0312413]

Kaplan, D. L., Kulkarni, S. R., van Kerkwijk, M. H., \& Marshall, H. L. 2002, ApJ, 570, L79

Kaplan, D. L., van Kerkwijk, M. H., Marshall, H. L., et al. 2003, ApJ, 590, 1008

Lai, D., \& Ho, W. C. G. 2003, ApJ, 588, 962

Link, B. 2003, in Radio Pulsars, ed. M. Bailes, D. Nice, \& S. E. Thorsett (San Francisco: ASP), ASP Conf. Ser., 302, 241

Motch, C., Zavlin, V. E., \& Haberl, F. 2003, A\&A, 408, 323

Paerels, F., Mori, K., Motch, C., et al. 2001, A\&A, 365, L298

Ruderman, M. 2003 [astro-ph/0310777]

Stairs, I. H., Lyne, A. G., \& Shemar, S. L. 2000, Nature, 406, 484

Strüder, L., Briel, U., Dennerl, K., et al. 2001, A\&A, 365, L18

van Kerkwijk, M. H. 2004, in Young neutron stars and their environments, ed. F. Camilo, \& B. Gaensler, ASP Conf. Ser. (San Francisco: ASP), in press [astro-ph/0310389]

Verner, D. A., Ferland, G. J., Korista, K. T., \& Yakovlev, D. G. 1996, ApJ, 465, 487

Wilms, J., Allen, A., \& McCray, R. 2000, ApJ, 542, 914 